\title{
Wave equation solution for multilayer planar waveguides in a spatial frequency domain
}

\author{
V.M. Fitio ${ }^{1}$, A.V. Bendzyak ${ }^{1}$, I.Y. Yaremchuk ${ }^{1}$, Y.V. Bobitski ${ }^{1,2}$ \\ ${ }^{1}$ Department of Photonics, Lviv Polytechnic National University, Lviv, Ukraine \\ ${ }^{2}$ Faculty of Mathematics and Natural Sciences, University of Rzeszów, Rzeszów, Poland
}

\begin{abstract}
Possibilities of new numerical method for solving the wave equation of multilayer planar waveguide were investigated. The method is based on the use of Fourier transform to the wave equation and its solution in the frequency domain by using the numerical method. The final task of finding propagation constants and the Fourier images of the fields in discrete form is reduced to the problem on the eigenvalues and eigenvectors. The new method provides highly accurate calculation of propagation constants and their fields and is characterized by high numerical stability.
\end{abstract}

Keywords: Fourier transform, multilayer planar waveguides, propagation constants.

Manuscript received 29.02.17; revised version received 03.10.17; accepted for publication 07.12.17; published online 07.12.17.

\section{Introduction}

The primary method of determining the propagation constants of localized modes in multilayer planar waveguides is based on solution of the transcendental equation [1], and this method is easy to use for a simple waveguide. However, modern integrated optics uses complex multilayer waveguides, for example, semiconductor lasers with double heterostructure, consists of five layers, and each layer has its own refractive index [2]. Distributed feedback waveguide microlaser, based on organic semiconductors has 3 or 4 layers [3, 4]. Increasing the number of layers complicates the transcendental equation and, therefore, its solution. If you have a simple waveguide in which there are two jumps of the refractive index (permittivity), the transcendental equation is defined as the equality of the $4 \times 4$ matrix determinant to zero [1], which is formed as equality of tangential components of the fields at the boundary between the layers. For the waveguide shown in Fig. 1 the transcendental equation is based on the $12 \times 12$ matrix.

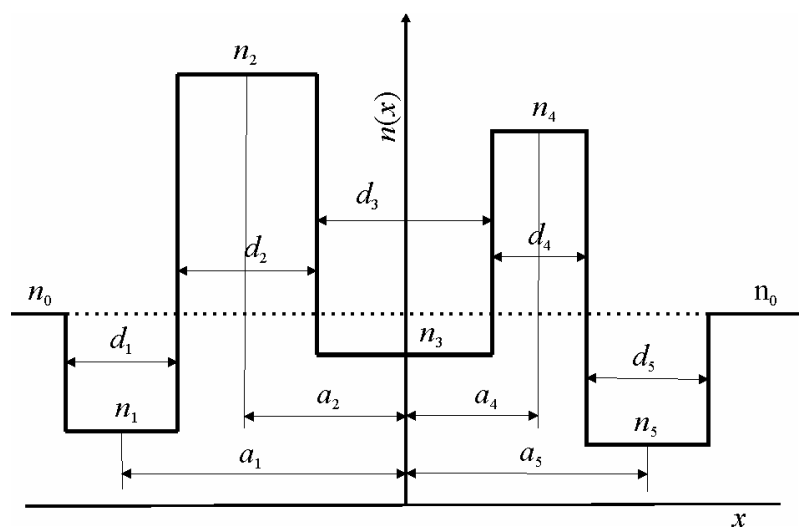

Fig. 1. Scheme of non-symmetric multilayer planar waveguide with six fixed values of refractive indices.

There is a well-known numerical method in coordinate area [5], in which the second derivative is replaced with differential operator and the calculation of propagation constants of waveguide modes and related fields is reduced to solution of the problem on eigenvalues and eigenvectors. However, this method does not 
ensure high accuracy $[5,6]$, also it is difficult to use it for TM polarization waves, as the function of the dielectric permittivity for such waveguide has a discontinuity of the first kind.

Thus, a numerical method to solve the wave equation for planar gradient waveguides in the frequency domain has been proposed [6,7]. Analyzed in these works are various known methods used for calculation of propagation constants of waveguide modes for gradient waveguides. All of these methods are mostly approximate, and only for few waveguides the calculations are exact [7], and they are based on solution of the wave equation in coordinate dimension. The proposed method $[6,7]$ is based on using Fourier transform to the wave equation, so as a result, one can get an integral equation in the frequency domain. Then, it is pertinent to write this equation in a discrete frequency spectrum by reducing it to solution of the well-known problem of higher algebra on eigenvalues and eigenvectors. The new method provides high accuracy and is characterized with a high numerical stability. It is also effective for symmetric and nonsymmetric waveguides and both for TE and TM wave polarizations [6].

It is shown below that this new method is also effective for multilayer planar waveguides. The example of such a waveguide is provided in Fig. 1.

\section{Theoretical foundations of the method for multilayer waveguides}

In compliance to the refractive index of each layer $n_{m}$, let's put permittivity $\varepsilon_{m}=n_{m}^{2}$, where $m$ varies from 0 to $M$. The thickness of each layer is equal to $d_{m}$, and it is shifted from $\mathrm{Y}$-axis by the distance $a_{m}$. The functional dependence of waveguide permittivity is expressed as:

$$
\varepsilon(x)=\varepsilon_{0}+\sum_{1}^{M-1} \delta \varepsilon_{m 0} \operatorname{rect}\left[\frac{1}{d_{m}}\left(x-a_{m}\right)\right]
$$

where $\delta \varepsilon_{m 0}=\varepsilon_{m}-\varepsilon_{0}$, and the function $\operatorname{rect}(x)$ is defined as:

$$
\operatorname{rect}(x)= \begin{cases}1, & \text { if }|x| \leq 1 / 2 \\ 0, & \text { if }|x|>1 / 2\end{cases}
$$

Fourier image of the function $\operatorname{rect}\left[\frac{1}{d_{m}}\left(x-a_{m}\right)\right]$, according to the scaling and displacement theorems is equal to [8]:

$$
F\left\{\operatorname{rect}\left[\frac{1}{d_{m}}\left(x-a_{m}\right)\right]\right\}=\frac{\sin \left(\pi u d_{m}\right)}{\pi u} \exp \left(-i 2 \pi u a_{m}\right),
$$

where $u$ in current situation is the spatial frequency.
Therefore, fourier image of the expression (1) is equal to:

$F\{\varepsilon(x)\}=\varepsilon_{0} \delta(u)+\sum_{m=1}^{M-1} \delta \varepsilon_{m 0} \frac{\sin \left(\pi u d_{m}\right)}{\pi u} \exp \left(-i 2 \pi u a_{m}\right)$

where $F\{\ldots\}$ is Fourier transform, $\delta(u)-$ Dirac delta function.

If the electric field of the waveguide mode is perpendicular to plane $x Z$ (TE polarization), then the corresponding wave equation can be expressed as[1]:

$$
\frac{d^{2} E(x)}{d x^{2}}+\left(\frac{2 \pi}{\lambda}\right)^{2} \varepsilon(x) E(x)=\beta^{2} E(x) .
$$

The functions $E(x)$ and $H(x)$ that describe electric fields in localized waveguide modes, are absolutely integrated, also, these functions and their first derivatives tend to zero, if $x \rightarrow \pm \infty$. Thus, Fourier transform can be applied to these functions, their first and second derivatives.

Besides, to the functions, for which Fourier transform exists, meaning $F\{G(x)\}=G(u)$, $F\{H(x)\}=H(u)$, the following expression is true:

$F\{G(x) H(x)\}=\int_{-\infty}^{\infty} G(u-v) H(v) d v$

Exp. (4) is known as convolution theorem [8].

After applying Fourier transform to right and left sides of equation (3), one can get the following expression:

$-4 \pi^{2} u^{2} E(u)+\left(\frac{2 \pi}{\lambda}\right)^{2} \int_{-\infty}^{\infty} \varepsilon(u-v) E(v) d v=\beta^{2} E(u)$,

where $\varepsilon(u)=\int_{-\infty}^{\infty} \varepsilon(x) \exp (-i 2 \pi u x) d x$.

As a result, from the differential equation (3) for eigenvalues and eigenvectors we obtained integral equation (5). In this equation, we can replace the integral with a sum. Therefore, after replacing continuous values of spatial frequencies $u$ and $v$ with discrete ones, we will get:

$$
\begin{aligned}
& -4 \pi^{2}\left(u_{s}\right)^{2} E\left(u_{s}\right)+\left(\frac{2 \pi}{\lambda}\right)^{2} \sum_{k=-(N-1) / 2}^{(N-1) / 2} \varepsilon\left(u_{s}-v_{k}\right) E\left(u_{v}\right) \Delta= \\
& =\beta^{2} E\left(u_{s}\right),
\end{aligned}
$$

where $\Delta=u_{\max } / N, u_{s}=s \Delta, v_{k}=k \Delta,-(N-1) / 2 \leq s$, $k \leq(N-1) / 2$. 
To solve this equation, $N$ should be sufficiently large and, for convenience, odd. Let's write the latter equation for a set of discrete frequencies $u_{s}=s \Delta$, where $s$ varies from $-(N-1) / 2$ to $(N-1) / 2$. As a result, we can write the set of these equations in quantity $N$ as a matrix equation. Also, $\beta^{2}$ should be common for every s:

$$
(\mathbf{P}+\mathbf{U}) \mathbf{E}=\beta^{2} \mathbf{E},
$$

where $\mathbf{P}$ - diagonal matrix, elements of which are equal to $-4(\pi s \Delta)^{2}, \mathbf{U}-$ symmetrical square matrix, elements of which are equal to $\left(\frac{2 \pi}{\lambda}\right)^{2} \varepsilon(s \Delta-k \Delta) \Delta, \mathbf{E}-$ rowvector, with elements $E(s \Delta)$.

Transition from the differential equation (3) to the integral equation (5), and also transition from the integral equation (5) to the matrix equation (6) is explained in details in $[6,7]$. It is worth to note, that solution to all the three equations leads to the problem of eigenvalues and eigenfunctions. However, only equations (5) and (7) are true for a discrete number of propagation constants of localized modes.

Respectively, the complications of calculating the propagation constants are reduced to the problem on eigenvalues (square of the propagation constant) and eigenvectors (discrete Fourier image $E(x)$ ), which corresponds to the relevant value $\beta$. We can have several eigenvalues and corresponding eigenvectors that are orthogonal. After applying inverse discrete Fourier transform to eigenvector, we will get the relevant electric field distribution $E(x)$.

\section{Example of numerical modeling}

Practical use of the proposed method will be demonstrated for multilayer planar waveguide, scheme of which was shown in Fig. 1, with the following parameters: $\lambda=1 \mu \mathrm{m}, \quad n_{0}=1.5, \quad n_{1}=1.4, \quad n_{2}=1.7$, $n_{3}=1.45, n_{4}=1.6, n_{5}=1.35, d_{1}=4 \mu \mathrm{m}, d_{2}=2 \mu \mathrm{m}$, $d_{3}=2 \mu \mathrm{m}, d_{4}=2 \mu \mathrm{m}, d_{5}=2 \mu \mathrm{m}, a_{1}=-5 \mu \mathrm{m}, a_{2}=-2 \mu \mathrm{m}$, $a_{3}=0, a_{4}=2 \mu \mathrm{m}, a_{5}=5 \mu \mathrm{m}$.

As it is known, the accuracy of calculation is defined by parameters of numerical process $N$ and $u_{\max }$. Propagation constants, calculated at $N=2001$ and $u_{\max }=$

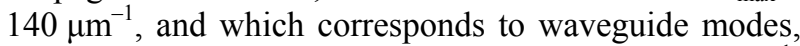
are, respectively, equal to: $\beta_{0}=10.59724925 \mu^{-1}$, $\beta_{1}=10.34367530 \mu \mathrm{m}^{-1}, \quad \beta_{2}=9.96936112 \mu \mathrm{m}^{-1}$, $\beta_{3}=9.91979424 \mu \mathrm{m}^{-1}, \beta_{4}=9.71932685 \mu \mathrm{m}^{-1}$.

Propagation constants calculated for the given waveguide are shown in Table 1. As we can see from Table 1, propagation constants are calculated for a wide range of $u_{\max }$, and the highest accuracy can be reached within the range $u_{\max }=120 \ldots 140$. Calculation accuracy in this range reaches 8-th decimal place, depending on the mode number. Besides, in Table 1, common numbers are highlighted for every calculated mode, depending on the value of $u_{\max }$.

In the case of non-symmetrical waveguide, dependences of calculated propagation constants from $u_{\max }$ at fixed values of $N=1001$ and $N=2001$ are shown in Figs. 1 and 2.

It follows from Fig. 2 that the change of $u_{\max }$ within the range from 10 up to $100 \mu \mathrm{m}^{-1}$ slightly affects accuracy of calculated propagation constants, and only calculations outside of this range leads to changes. When $N=2001$ (see Fig. 3), within the range of $u_{\max }$ from 10 up to $200 \mu \mathrm{m}^{-1}$, the calculated propagation constants are actually stable.

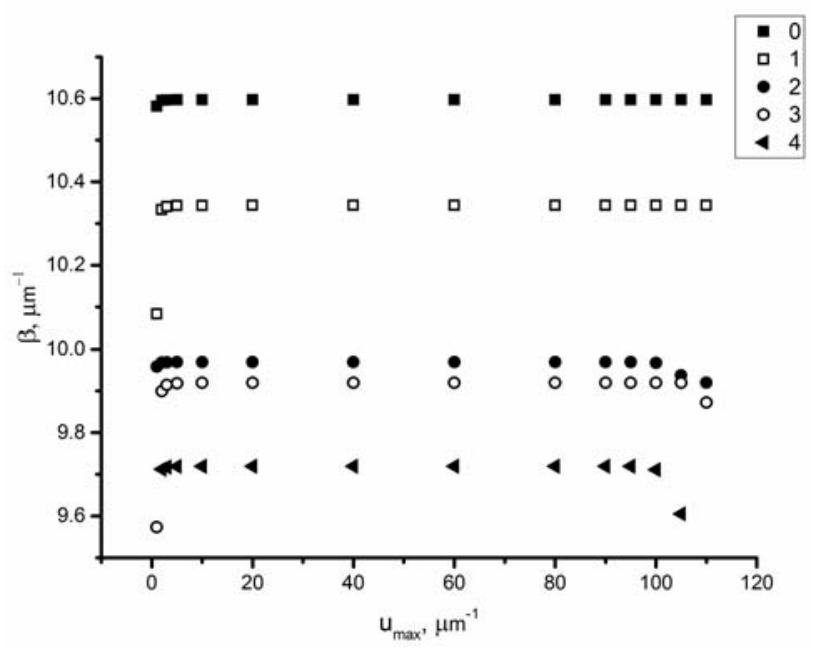

Fig. 2. Dependence of calculated propagation constants from $u_{\max }$ at $N=1001$. Digits near calculation dots indicate the mode number.

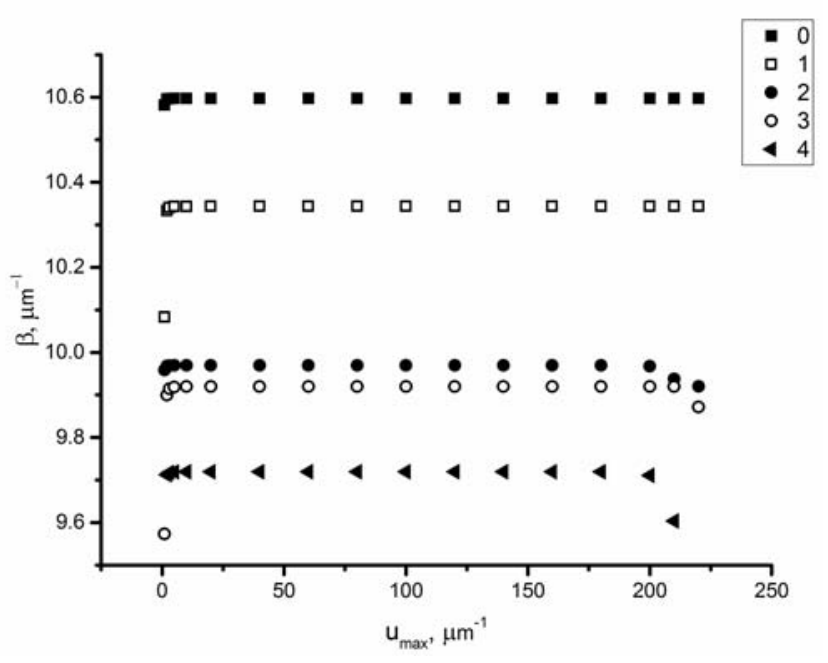

Fig. 3. Dependence of calculated propagation constants from $u_{\max }$ at $N=2001$. Digits near calculation dots indicate the mode number. 
Table 1. Calculated propagation constants for a non-symmetric waveguide $(N=2001)$.

\begin{tabular}{|l|l|l|l|l|l|l|}
\hline$u_{\max }$ & $60 \mu \mathrm{m}^{-1}$ & $80 \mu \mathrm{m}^{-1}$ & $100 \mu \mathrm{m}^{-1}$ & $120 \mu \mathrm{m}^{-1}$ & $140 \mu \mathrm{m}^{-1}$ & $160 \mu \mathrm{m}^{-1}$ \\
\hline$\beta_{0}$ & $\mathbf{1 0 . 5 9 7 2 4 9 1 7}$ & $\mathbf{1 0 . 5 9 7 2 4 9 2 2}$ & $\mathbf{1 0 . 5 9 7 2 4 9 2 3}$ & $\mathbf{1 0 . 5 9 7 2 4 9 2 4}$ & $\mathbf{1 0 . 5 9 7 2 4 9 2 4}$ & 10.59724925 \\
\hline$\beta_{1}$ & $\mathbf{1 0 . 3 4 3 6 7 5 0 1}$ & $\mathbf{1 0 . 3 4 3 6 7 5 1 8}$ & $\mathbf{1 0 . 3 4 3 6 7 5 2 5}$ & $\mathbf{1 0 . 3 4 3 6 7 5 2 8}$ & $\mathbf{1 0 . 3 4 3 6 7 5 2 9}$ & $\mathbf{1 0 . 3 4 3 6 7 5 3 0}$ \\
\hline$\beta_{2}$ & $\mathbf{9 . 9 6 9 3 6 1 0 7 6}$ & $\mathbf{9 . 9 6 9 3 6 1 1 0 6}$ & $\mathbf{9 . 9 6 9 3 6 1 1 1 6}$ & $\mathbf{9 . 9 6 9 3 6 1 1 2 1}$ & $\mathbf{9 . 9 6 9 3 6 1 1 2 3}$ & $\mathbf{9 . 9 6 9 3 6 1 1 2 5}$ \\
\hline$\beta_{3}$ & $\mathbf{9 . 9 1 9 7 9 3 6 0 5}$ & $\mathbf{9 . 9 1 9 7 9 4 0 0 3}$ & $\mathbf{9 . 9 1 9 7 9 4 1 4 4}$ & $\mathbf{9 . 9 1 9 7 9 4 2 0 7}$ & $\mathbf{9 . 9 1 9 7 9 4 2 3 8}$ & $\mathbf{9 . 9 1 9 7 9 4 2 5 6}$ \\
\hline$\beta_{4}$ & $\mathbf{9 . 7 1 9 3 2 6 6 6 5}$ & $\mathbf{9 . 7 1 9 3 2 6 7 8 2}$ & $\mathbf{9 . 7 1 9 3 2 6 8 2 4}$ & $\mathbf{9 . 7 1 9 3 2 6 8 4 2}$ & $\mathbf{9 . 7 1 9 3 2 6 8 5 2}$ & $\mathbf{9 . 7 1 9 3 2 6 8 5 7}$ \\
\hline
\end{tabular}

Comparison of these two plots shows that lower limit $u_{\max }$ doesn't change significantly with a change of $N$, although the upper limit is rising twice.

Therefore, we can conclude that lower limit $u_{\max }$ is independent of $N$, and this bottom limit can be determined by a criterion, that is described and wellgrounded in the paper [7]. The upper limit $u_{\max }$ increases in proportional to $N$, which defines the maximum value $\Delta=u_{\max } / N$, consistent with the sample theorem [8], as $\Delta=u_{\max } / N$, and this value defines a range in the coordinate region $[-0.5 / \Delta, 0.5 / \Delta]$, where the fields of modes are being calculated. At the edges of this range, electrical fields of discrete modes are assumed to be equal to zero.

The electrical field distributions for the first three modes are shown in Fig. 4. Computational process parameters are equal to $N=2001$ and $u_{\max }=120 \mu \mathrm{m}^{-1}$.

It follows from Fig. 4 that modes with indices 0 and 1 , are basically concentrated in the layer with the refractive index $n_{2}=1.7$, the mode with the index 3 is concentrated in the layer with $n_{4}=1.6$. Fields for the latter two modes are shown in Fig. 5. The numerical process parameters are the same as for the first three modes. The electrical field of the mode with the index 3 is concentrated in the layer with $n_{2}=1.7$, and field of the mode with the index 4 is concentrated in the layer with $n_{4}=1.6$. This is related with the fact that the respective layers have the highest refractive indices, and the layer 3 between them has the refractive index $n_{3}=1.45$, which is less than $n_{0}=1.5$. Therefore, this layer is of some kind of potential barrier for tunneling photons from the layer 2 to the layer 4, and vice versa.

In the case, when refractive index of the mode 3 will be higher than $n_{0}$, the waveguide modes will spread over three layers, especially it is typical for the modes of higher indices. A similar result will be observed, when the thickness of the third layer $d_{3}$ will be significantly reduced.

In addition, we calculated propagation constants for symmetrical multilayer planar waveguide with a profile shown in Fig. 6. Parameters of calculated waveguide are as follows: $\lambda=1 \mu \mathrm{m}, n_{0}=1.45, n_{1}=1.47, n_{2}=1.45$, $n_{3}=1.5, n_{4}=1.45, n_{5}=1.47, d_{1}=2 \mu \mathrm{m}, d_{2}=2.5 \mu \mathrm{m}$, $d_{3}=1.5 \mu \mathrm{m}, d_{4}=2.5 \mu \mathrm{m}, d_{5}=2 \mu \mathrm{m}, a_{1}=-4.25 \mu \mathrm{m}$, $a_{2}=-2 \mu \mathrm{m}, a_{3}=0, a_{4}=2 \mu \mathrm{m}, a_{5}=4.25 \mu \mathrm{m}$.

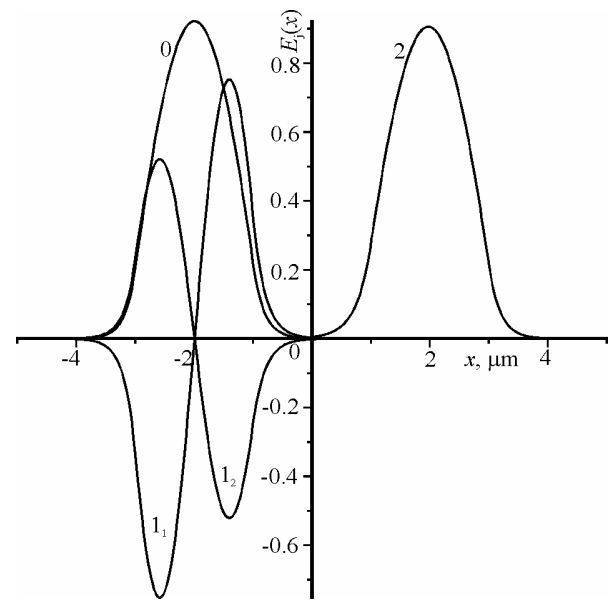

Fig. 4. Field distribution in waveguide for the first three modes. The field with the index 1 is $\left(1_{1}-\right.$ real part, $1_{2}-$ imaginary part).

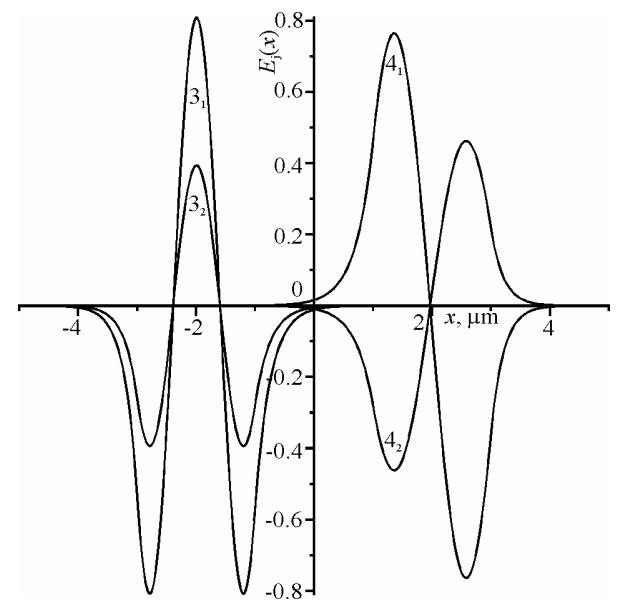

Fig. 5. Field distribution in the waveguide for the last two modes.

Since our waveguide structure is spatially symmetrical, the electrical field distribution may be either symmetric or antisymmetric. Its fields can be described by real functions: modes with even indices will correspond to fields that are defined by the symmetrical function $E_{j}(x)=E_{j}(-x)$, and modes with odd indices are defined by the following expression:

$$
E_{j}(x)=-E_{j}(-x) \text {. }
$$




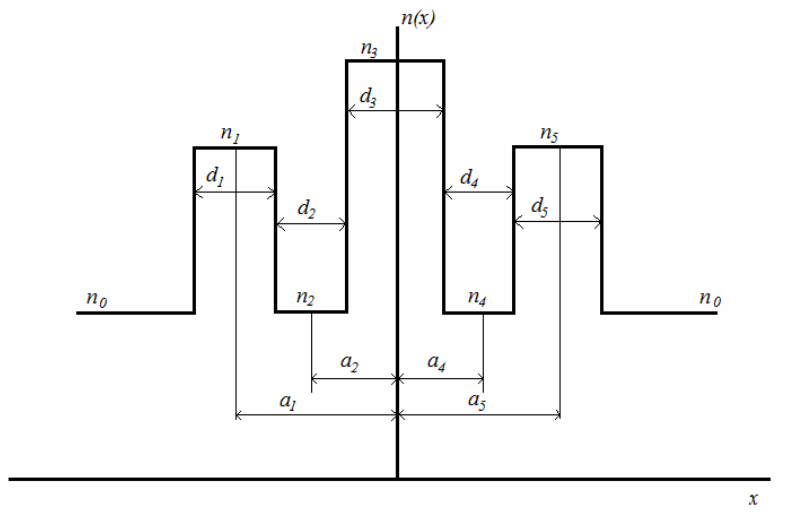

Fig. 6. Scheme of symmetrical multilayer planar waveguide with six fixed values of refractive indices.

As it was said previously, calculation accuracy is defined by the parameters $N$ and $u_{\max }$. When calculation parameters were $N=2001$ and $u_{\max }=60 \mu \mathrm{m}^{-1}$, we obtained the following values of propagation constants: $\beta_{0}=9.33165605 \mu \mathrm{m}^{-1}, \quad \beta_{1}=9.19041624 \mu \mathrm{m}^{-1}$, $\beta_{2}=9.19029113 \mu \mathrm{m}^{-1}, \quad \beta_{2}=9.19029113 \mu \mathrm{m}^{-1}$, $\beta_{3}=9.12237133 \mu \mathrm{m}^{-1}$.

In Figs. 7 and 8 one can see the dependences of calculated propagation constants from $u_{\max }$ at fixed values of $N=1001$ and $N=2001$.

We can observe from Fig. 7 that propagation constants within the range of $u_{\max }$ from 10 up to $80 \mu \mathrm{m}^{-1}$ practically do not change, but, with increase of the top limit, we can observe some kind of divergence between the 1-st and 2-nd propagation constants.

As in the case with non-symmetrical waveguide, increasing $N$ twice leads to widening $u_{\max }$ range. Here, as we can see from Fig. 8 , the range of $u_{\max }$ is from 10 up to $160 \mu^{-1}$, and outside of this range we can also observe divergence of the 1-st and 2-nd modes. It means that in the point of divergence between these two modes, we have the maximum permissible value $\Delta=u_{\max } / N$. This condition agrees with the sampling theorem [8], as $x_{\max }=1 / \Delta$, and this value defines the range in coordinate dimensions $[-0.5 / \Delta, 0.5 / \Delta]$, in which the mode fields are calculated, and at the edges of this range, electrical fields of discrete modes should be equal to zero, which can be observed in Fig. 9.

In Table 2, one can see the calculated propagation constants for a symmetrical waveguide. Analyzing these data, we can see that within the range of $u_{\max }=60 \ldots 80 \mu \mathrm{m}^{-1}$ we have the highest accuracy (up to 8 digits). In addition, the mode with the index 0 , both for symmetrical and non-symmetrical waveguides is the most accurate. With increase of the mode index, accuracy decreases, and is the lowest for the last mode. These results agree with conclusion, described in the paper [7]. With further increase of $u_{\max }$, the last mode disappears, and instead of four eigenvalues of matrix equation Eq. (6), we will have three eigenvalues.

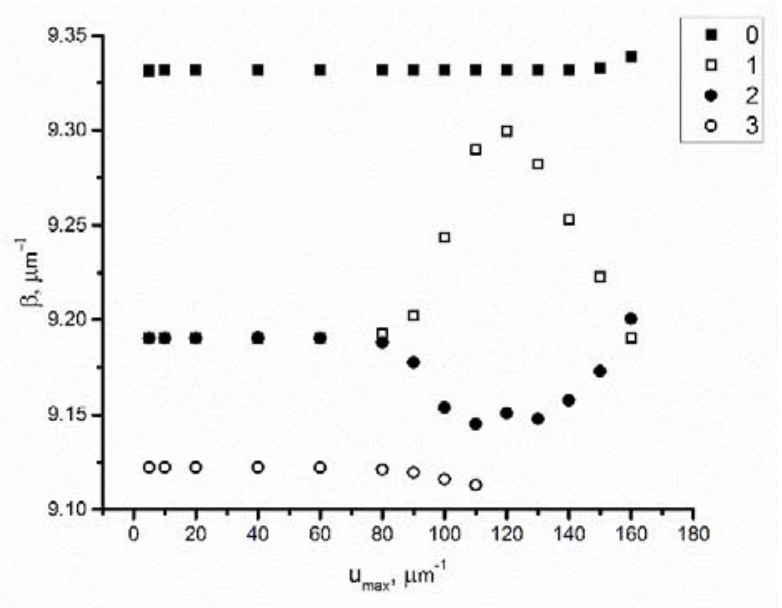

Fig. 7. Dependence of calculated propagation constants from $u_{\max }$ at $N=1001$.

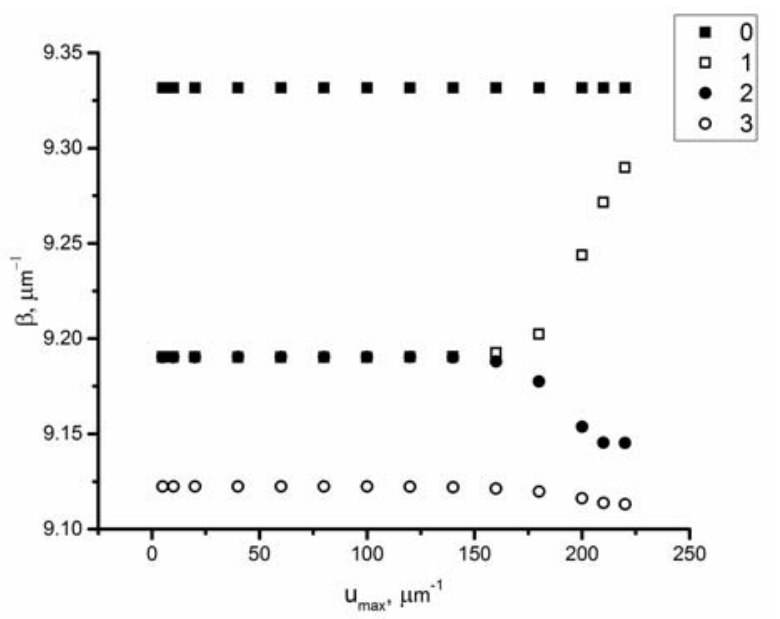

Fig. 8. Dependence of calculated propagation constants from $u_{\max }$ at $N=2001$.

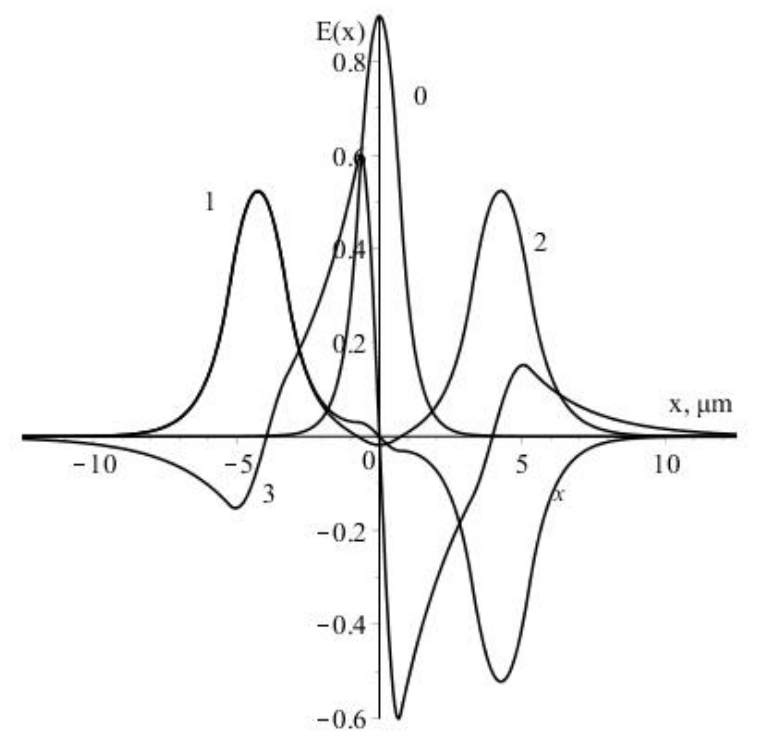

Fig. 9. Field distribution in the waveguide for all the waveguide modes, $N=2001, u_{\max }=60 \mu \mathrm{m}^{-1}$. 
Table 2. Calculated propagation constants for a symmetric waveguide $(N=2001)$.

\begin{tabular}{|l|l|l|l|l|l|l|}
\hline$u_{\max }$ & $20 \mu \mathrm{m}^{-1}$ & $40 \mu \mathrm{m}^{-1}$ & $60 \mu \mathrm{m}^{-1}$ & $80 \mu \mathrm{m}^{-1}$ & $100 \mu \mathrm{m}^{-1}$ & $120 \mu \mathrm{m}^{-1}$ \\
\hline$\beta_{0}$ & $\mathbf{9 . 3 3 1 6 5 5 6 9}$ & $\mathbf{9 . 3 3 1 6 5 6 0 1}$ & $\mathbf{9 . 3 3 1 6 5 6 0 5}$ & $\mathbf{9 . 3 3 1 6 5 6 0 5}$ & $\mathbf{9 . 3 3 1 6 5 6 0 6}$ & $\mathbf{9 . 3 3 1 6 5 6 0 6}$ \\
\hline$\beta_{1}$ & $\mathbf{9 . 1 9 0 2 9 1 0 9}$ & $\mathbf{9 . 1 9 0 2 9 1 1 3}$ & $\mathbf{9 . 1 9 0 2 9 1 1 3}$ & $\mathbf{9 . 1 9 0 2 9 1 1 4}$ & $\mathbf{9 . 1 9 0 2 9 1 4 1}$ & $\mathbf{9 . 1 9 0 3 0 6 4 3}$ \\
\hline$\beta_{2}$ & $\mathbf{9 . 1 9 0 4 1 6 1 9}$ & $\mathbf{9 . 1 9 0 4 1 6 2 3}$ & $\mathbf{9 . 1 9 0 4 1 6 2 4}$ & $\mathbf{9 . 1 9 0 4 1 6 2 4}$ & $\mathbf{9 . 1 9 0 4 1 5 9 7}$ & $\mathbf{9 . 1 9 0 4 0 1 1 3}$ \\
\hline$\beta_{3}$ & $\mathbf{9 . 1 2 2 3 7 0 8 5}$ & $\mathbf{9 . 1 2 2 3 7 1 3 4}$ & $\mathbf{9 . 1 2 2 3 7 1 3 3}$ & $\mathbf{9 . 1 2 2 3 6 8 8 0}$ & $\mathbf{9 . 1 2 2 3 4 4 8 7}$ & $\mathbf{9 . 1 2 2 2 4 3 0 7}$ \\
\hline
\end{tabular}

From Figs. 7 and 8, and from Table 2, we can see that propagation constants with the indices 1 and 2 are very close. We cannot distinguish them visually from Figs. 7 and 8, but analyzing Table 2, we can conclude that they differ in the fifth significant digit. This is because the energy of this waveguide mode is concentrated in two identical outer layers with the refractive indices $n_{1}$ and $n_{5}$, which are equal.

Analyzing Fig. 9, we can note that the mode with the index 0 concentrates in the central layer, modes with indices 1 and 2 - in outer layers, and the last mode - at the edges of central layer. As noted above, since the waveguide is spatially-symmetrical, the modes with indices 0 and 2 are symmetrical with respect to vertical axes, and the modes with the indices 1 and 3 are antisymmetric.

\section{Conclusions}

Results of this paper show that using the proposed numerical method to solve multilayer planar waveguide gives accurate results. Propagation constants and distribution of electric fields for symmetric and antisymmetric waveguide have been calculated. Optimal parameters for propagation constants calculations have been obtained, namely: $u_{\max }$ and $N$. We have found that the lower limit of $u_{\max }$ does not change significantly with a change of $N$, and upper limit is 2-fold increased, while increasing $N$ twice. We can conclude that the lower limit of $u_{\max }$ is independent of $N$, and the upper limit of $u_{\max }$ increases in proportion to $N$. In other words, $u_{\max }$ defines the maximum allowed value of $\Delta=u_{\max } / N$, which is consistent with the sampling theorem [8], as $x_{\max }=1 / \Delta$, and this value defines the range in the coordinate region $[-0.5 / \Delta, 0.5 / \Delta]$, in which the electrical fields are calculated, and at the edges of this range the electrical fields of discrete modes are practically equal to zero.

The obtained results are significant for development of DFB waveguide lasers, and also for physical measurement sensors that are based on the principle of disorder of resonance of waveguide modes. Knowing the propagation constants in a waveguide, and corresponding fields, enables us to calculate gain coefficients of active laser media, binding coefficients between modes, and also to explain characteristics of similar multilayer waveguide lasers with Bragg gratings [3]. The calculated fields show that they approach their maximum value in layers with a higher refractive index. This conclusion is also valuable for development of multilayer waveguide lasers. Since the probabilities of laser transitions are in proportion to the field powered two, it is efficient to make the active layers with a high refractive index.

\section{References}

1. Unger H.G. Planar optical waveguides and fibres. Oxford Engineering Science Series. Oxford, Clarendon Press, 1977, P. 97.

2. Yariv A. Quantum Electronics, 2nd. ed. John Wiley \& Sons, New York, NY, 1975, P. 123.

3. Smirnova T.N., Sakhno O.V., Stumpe J., Janin J., Fitio V.M. Polymer distributed feedback dye laser with an external volume Bragg grating inscribed in a nanocomposite by holographic technique. JOSA B. 2016. 33, No. 2. P. 202-210.

4. Smirnova T.N., Sakhno O.V., Fitio V.M., Gritsai Y. and Stumpe J. Simple and high performance DFB laser based on dye-doped nanocomposite volume gratings. Laser Phys. Lett. 2014. 11. P. 125804-125811.

5. Rganov A.G., Grigas S.E. Numerical algorithm for waveguide and leaky modes determination in multilayer optical waveguides. Techn. Phys. 2010. 55. P. 1614-1618.

6. FitioV .M., Romakh V.V., Bobitski Y.V. Numerical method for analysis of waveguide modes in planar gradient waveguides. Material Science (Medžiagotyra). 2014. 20, No 3. P. 256261.

7. Fitio V.M., Romakh V.V., Bartkiv L.V., Bobitski Y.V. The accuracy of computation of mode propagation constants for planar gradient waveguides in the frequency domain. Materials Science \& Engineering Technology (Materialwissenschaft und Werkstofftechnik). 2016. 47, No. 2-3. P. 237-245.

8. Goodman J.W. Introduction to Fourier Optics. San Francisco, Mcgraw-Hill Book Company, 1968. P. 8. 